Genetic:

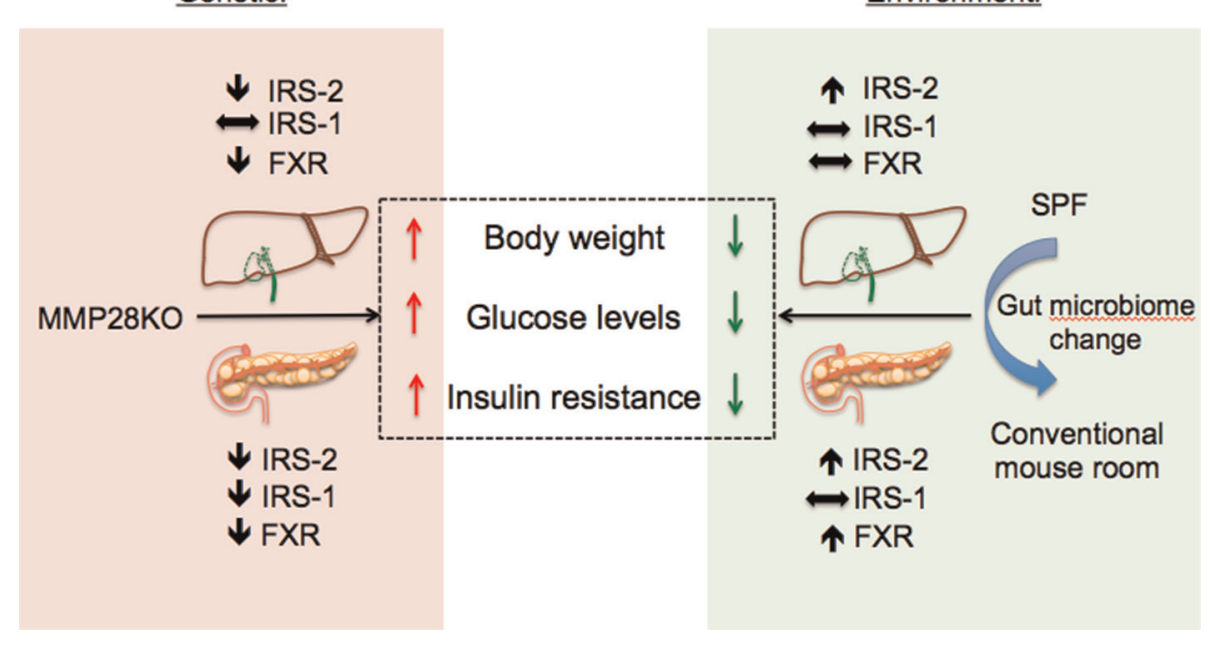

Environment:
Conclusion In conclusion, the MMP28 gene knockout effect and the change of gut microbiome upon changing living environments influence the metabolic profile through modulations in the insulin and FXR signalling pathways.

\section{PTU-008 DIABETES MELLITUS IN PATIENTS WITH AUTOIMMUNE HEPATITIS (AIH): AT DIAGNOSIS AND FOLLOWING PREDNISOLONE TREATMENT}

Selena Dixon*, Laura Harrison, Barbara Hoeroldt, Elaine McFarlane, Dermot Gleeson. Liver Unit, Sheffield Teaching Hospitals NHS Foundation Trust, Sheffield, UK

\subsection{6/gutjnl-2019-BSGAbstracts.217}

Introduction Patients receiving corticosteroids are at increased risk of new-onset diabetes mellitus (NODM), related to steroid dose and duration and which may persist despite steroid withdrawal. Treatment of Autoimmune Hepatitis (AIH) remains corticosteroid-based, yet there are few data on NODM in patients with AIH. We sought to assess the incidence, time course and risk factors for NODM in patients with $\mathrm{AIH}$, treated with prednisolone.

Methods Retrospective single-centre audit of patients with AIH (1999 IAHG criteria), presenting 1987-2016. We collected data on age, gender, BMI, ethnicity, diagnosis and treatment of diabetes, corticosteroid dose/duration and presence of cirrhosis at AIH diagnosis. NODM was defined according to World Health Organization criteria or commencement of drug treatment for diabetes mellitus.

Results Of 309 informative patients (80\% women, 92\% White British, age (mean) 55 years and BMI 28 at AIH diagnosis), $30(10 \%)$ already had diabetes mellitus at diagnosis of $\mathrm{AIH}$ (Type 2 in 27, Type 1 in 3); these were older than those without diabetes (mean+SD $62 \pm 15$ vs $53 \pm 19$ years; $\mathrm{p}=0.013)$ and had higher BMI $(30 \pm 7$ vs $27 \pm 6 ; \mathrm{p}=0.07)$. Of the remaining 279 patients, 254 were treated with prednisolone, of which $49(19 \%)$ subsequently developed NODM (Type 2 in 47, Type 1 in 2). Rate of NODM development was $7 \pm 2 \%, 10 \pm 2 \%, 13 \pm 2 \%, 16 \pm 2 \%$ and $21 \pm 3 \%$ after 3 , $12,36,60$ and 120 months respectively. In 25 non-diabetic patients who did not receive prednisolone (1 received budesonide, 24 untreated), rate of NODM was lower (1 patient, 14 years after AIH diagnosis; $\mathrm{p}=0.08$ ).
Patients who developed NODM following prednisolone were older (mean + SD $55+14$ vs $53+18$ years; $\mathrm{p}=0.006)$, more overweight (BMI $30 \pm 7$ vs $27 \pm 5 ; \mathrm{p}=0.019$ ) at $\mathrm{AIH}$ diagnosis and were more likely to be Asian or African-Caribbean (9/49 vs $12 / 205$ patients) ethnicity than those that did not do so. Independent risk factors for developing NODM on Cox multivariate analysis were: higher BMI at AIH diagnosis $(p=0.001)$; Asian or African-Caribbean ethnicity $(p=0.003)$; older age at commencing prednisolone treatment $(\mathrm{p}=0.008)$ and initial dose of prednisolone $\geq 40 \mathrm{mg} /$ day $(\mathrm{p}=0.038)$. For NODM developing within 12 months, cirrhosis was also a risk factor $(p=0.013)$, along with Asian or African-Caribbean ethnicity and initial prednisolone dose $\geq 40 \mathrm{mg} /$ day.

Of 42 patients followed up, NODM resolved in only 1 of 20 following prednisolone discontinuation and in 3 patients in whom prednisolone dose was reduced.

Conclusions NODM is common in patients with prednisolonetreated AIH; risk factors include older age, obesity, Asian or African-Caribbean ethnicity and high initial prednisolone dose. NODM usually persists following prednisolone reduction or withdrawal. In patients with $\mathrm{AIH}$ and risk factors for NODM, high-dose prednisolone should be used with caution.

\section{PTU-009 LONG-TERM EFFECTS OF OBETICHOLIC ACID IN PATIENTS WITH PRIMARY BILIARY CHOLANGITIS ON INFLAMMATORY MARKERS}

${ }^{1}$ Gideon M Hirschfield, ${ }^{2}$ Frederik Nevens, ${ }^{3}$ Mitchell L Shiffman, ${ }^{4}$ Joost PH Drenth* ${ }^{5}$ Christopher L Bowlus, ${ }^{6}$ Victor Vargas, ${ }^{7}$ Pietro Andreone, ${ }^{8}$ Stephen Ryder, ${ }^{9}$ Alexander Liberman, ${ }^{9}$ Elizabeth Smoot Malecha, ${ }^{9}$ Leigh MacConell, ${ }^{10}$ Michael Trauner. ${ }^{1}$ Toronto Centre for Liver Disease, University of Toronto, Toronto, Canada; ${ }^{2}$ University Hospital KULeuven, Leuven, Belgium; ${ }^{3}$ Bon Secours Liver Institute of Hampton Roads, Newport News, USA; ${ }^{4}$ Radboudumc, Nijmegen, Netherlands; ${ }^{5}$ University of California, Davis, Sacramento, USA; ${ }^{6}$ Hospital Vall d'Hebron, Universitat Autònoma de Barcelona, Barcelona, Spain; ${ }^{7}$ University of Bologna, Bologna, Italy; ${ }^{8}$ NIHR Nottingham Biomedical Research Centre at Nottingham University Hospitals NHS Trust, Queen's Medical Centre, Nottingham, UK; ${ }^{9}$ Intercept Pharmaceuticals, Inc., San Diego, USA; ${ }^{10}$ Division of Gastroenterology and Hepatology, Medical University of Vienna, Vienna, Austria

\subsection{6/gutjnl-2019-BSGAbstracts.218}

Introduction Obeticholic Acid (OCA) is a potent and selective farnesoid $\mathrm{X}$ receptor agonist approved for the treatment of primary biliary cholangitis (PBC). $\mathrm{PBC}$ is a chronic liver 